\title{
Evaluación de la dinámica urbana para la ciudad de Envigado
}

Urban dynamics evaluation of Envigado city

\author{
Alejandro Betancourt ${ }^{\star}$ \\ Luis Antonio Quintero**
}

Fecha de recepción: 07/10/2011

Fecha de aprobación: 17/11/2011

Ingeniero Matemático, Research in Spatial Ecomics (RiSE group), Universidad EAFIT, Colombia. Correo electrónico: abetan16@eafir.edu.co

** PhD. Físico y Economista, Docente del Departamento de Ciencias Básicas, Universidad EAFIT, Colombia. Correo electrónico: Iquinte3@eafit.edu.co 
Evaluación de la dinámica urbana para la ciudad de Envigado

ALEJANDRO BETANCOURT

LUIS ANTONIO QUINTERO

\title{
Resumen
}

En este artículo se presenta la conceptualización metodológica y los principales resultados de un modelo de dinámica de sistemas, cuyo objetivo principal es soportar la toma de decisiones para la ciudad de Envigado en políticas de vivienda. Para esto se elaboró un modelo basado en escenarios, el cual mediante una combinación de la perspectiva del Producto Interno Bruto (PIB) y una estrategia de permisos de construcción modela la evolución aproximada de la demanda y la oferta de vivienda en la ciudad. Usando este modelo se obtienen algunos resultados importantes en función del escenario utilizado, por ejemplo se encuentra que la oferta de vivienda alcanzará el punto de saturación entre el 2040 y el 2046, situación ocasionada por la disponibilidad de suelos. Por último es importante resaltar que este artículo es una muestra de cómo herramientas académicas como la dinámica de sistemas pueden ayudar a los gobernantes en su labor de toma de decisiones.

\section{Palabras clave:}

Construcción, demografía, dinámica urbana, inmigración, migración, Plan de Ordenamiento Territorial (POT).

\begin{abstract}
This article presents the methodological conceptualization and the main results of a System Dynamics model, which main objective is to support the housing policies in the city of Envigado. The used methodology developed a scenario-based model to emulate the approximate evolution of the housing demand and supply for the city, using a scenario of the Gross Domestic Product (GDP) and a housing authorization strategy as input. Diverse results were obtained, for instance it was found that due to the soil availability, the housing supply reaches the saturation point between 2040 and 2046. Finally this article could be considered as an example of how academic tools such as System Dynamics can be used by decisions makers in the government.
\end{abstract}

\section{Key words:}

Construction, Demography,Urban Dynamics, Inmigration, Migration, TerritorialOrderingPlan.

Clasificación JEL: R00, R52, R58 


\section{Introducción}

Durante los últimos años el crecimiento de la población y de la oferta de vivienda se ha convertido en un problema de gran cuidado, especialmente en los países en desarrollo, debido a que es en la población donde se encuentra el alma de las ciudades. Por esta razón se han venido aplicando nuevas herramientas para ayudar a tomar decisiones correctas frente a las problemáticas que se desprenden de la dinámica poblacional.

En este caso se desarrolló un modelo, mediante la metodología de dinámica de sistemas, que representa la evolución de la población en el municipio de Envigado, con el fin de hacerle un seguimiento y proyectar su crecimiento de acuerdo con los patrones que se han dado en los últimos años.

\section{Descripción del problema}

El problema desarrollado por este modelo analiza la dinámica urbana en Envigado -Antioquia- y estudia la demanda y oferta de vivienda que se ha presentado en el municipio. Cabe anotar que Envigado, en los últimos veinte años, ha invertido gran cantidad de recursos en el desarrollo de nuevas viviendas, para albergar la afluencia de personas que migran hacia allí desde diversos sitios del valle del Aburrá en búsqueda de mejor calidad de vida y seguridad, lo cual ha convertido al municipio en uno de los de mayor crecimiento del país (Otra Parte).

El nivel de demanda de vivienda que se ha presentado en los últimos años es consecuencia directa de los buenos indicadores de seguridad y de calidad de vida de Envigado. Cifras oficiales muestran que aproximadamente el $98 \%$ de las personas que habitan las zonas urbanas cuentan con los servicios públicos básicos (Anuario estadístico del municipio de Envigado), lo que lo convierte en un municipio con alto nivel migratorio y atraer gran cantidad de población de Medellín, ciudad esta última que, de acuerdo con el Censo general de población, en el año 2005 contaba con 2.219 .861 habitantes (DANE). La demanda de vivienda también se ve afectada en una gran medida por el crecimiento autónomo del municipio de Envigado, es decir, por el aumento de población ocasionado por su tasa de nacimientos. 
Evaluación de la dinámica urbana para la ciudad de Envigado

ALEJANDRO BETANCOURT

LUIS ANTONIO QUINTERO

La atención de la demanda del suelo urbano es una tarea predominante para los alcaldes, lo cual torna este trabajo más complejo al considerar que gran cantidad de las tierras del municipio no son aptas para construcción, por sus inclinadas pendientes. Por esta razón, en el Plan de Ordenamiento Territorial (POT) municipal se limita de manera considerable el área apta para la construcción de nuevas viviendas. Además, si se piensa que a largo plazo el problema se complica aún más, ya que a pesar de que actualmente el municipio cuenta con una gran disponibilidad para construcción (v. figura 1), se prevé que dentro de cuarenta o cincuenta años esta disponibilidad será mucho menor, o posiblemente nula. Por dicha razón es de suma importancia planear el horizonte de desarrollo de las áreas disponibles y hacer previsiones para el momento en el que las tierras estén utilizadas en su totalidad.

Figura 1.

Densidad urbana definida en el Plan de Ordenamiento Territorial. ${ }^{1}$

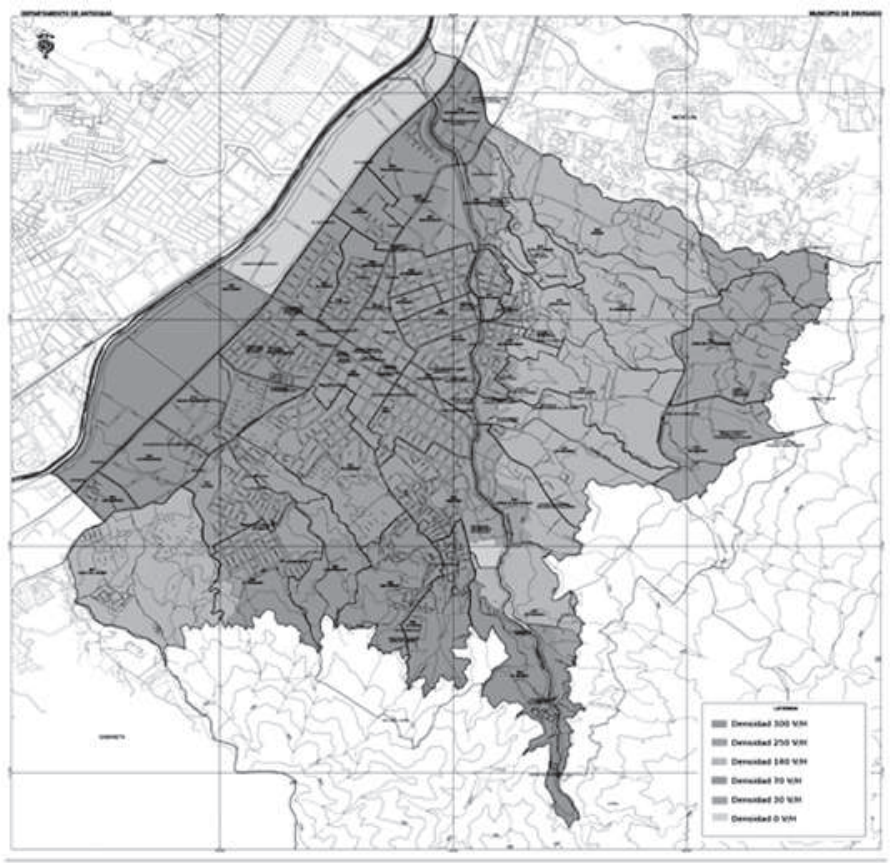

1 Mapa tomado de los planos del Plan de Ordenamiento Territorial; elaborado por la Oficina Asesora de Planeación. 


\section{Antecedentes metodológicos}

Desde finales de la década de los sesenta uno de los problemas a los que se destinó la dinámica de sistemas, después de las aplicaciones corporativas, fue a la dinámica urbana, con el trabajo pionero de Forrester (1969), el cual generó discusión en torno a esta propuesta, para entonces novedosa pero que fue acogida por parte de la comunidad académica, haciéndole una integración crítica (Harveya \& Roberth, 1970; Gray, Ressel \& Vararja, 1972; Ingram \& Kain, 1972; Saxton, 1979).

Debido a la complejidad y a la gran cantidad de variables que se pueden encontrar para el modelamiento adecuado de una ciudad, la metodología de dinámica de sistemas ofrece grandes ventajas, ya que permite representar y analizar el sistema desde sus elementos esenciales y estructurales. Por esta razón, en los últimos tiempos se han venido construyendo modelos que desarrollan y estudian problemas relacionados con la dinámica urbana de las ciudades, de modo que brinda un invaluable apoyo a los entes gubernamentales en los procesos de planeación. Se tienen ejemplos de aplicación en los casos de la ciudad de Seúl (Corea), donde se analiza su crecimiento autosuficiente, considerando los problemas que se le oponen como la saturación del transporte, el crecimiento de la infraestructura y los problemas ambientales (Moonseo et al., 2011, pp. 1-12); el modelo desarrollado para la ciudad de Beijin (China), que plantea la expansión de la ciudad hasta el 2020, en función de los factores limitantes que determinarían este crecimiento (Chunyang, Norio, Qiaofeng, Peijun \& Jingshui, 2006, pp. 323-345); el estudio de la disposición de desechos en la ciudad de New Jersey (Kollikkathara, 2009); el estudio de la ciudad de Puerto Aura en la región del lago Valsequillo, en Puebla (México), referido al sistema urbano, integrando diferentes dimensiones para dar cuenta de la dinámica del desarrollo sostenible (Durán-Encalada \& Paucar-Cáceres, 2009, pp. 77-99). La dinámica de sistemas también ha sido aplicada para planear el futuro de la ciudad costera española de Mataró, donde el modelo construido permitió brindar información a entidades gubernamentales españolas en torno a la evolución demográfica y geográfica de esta región (Reverté, 2003).

Asimismo, en diversos estudios aplicados se ha integrado la dinámica de sistemas con otras metodologías, como los sistemas de información geográficos (SIG), como en el caso del citado estudio para la ciudad de New Jersey (Kollikkathara, 2009), o el desarrollado en la ciudad de Chongqing (China) (Guan, Gao, Su, Li \& Hokao, 2011, pp. 1333-1344). 
Evaluación de la dinámica urbana para la ciudad de Envigado

ALEJANDRO BETANCOURT

LUIS ANTONIO QUINTERO

Por otra parte, se da la integración con otras metodologías como los modelos de autómatas celulares, en el caso de la ciudad de Beijin (Chunyang, Norio, Qiaofeng, Peijun \& Jingshui, 2006, pp. 323-345). En varios trabajos de modelación urbana se ha integrado la dinámica de sistemas con modelos de redes neuronales para extracción de la dinámica de variables del sistema, como las económicas, energéticas y ambientales, y la optimización de las trayectorias resultantes en los escenarios analizados, mediante la aplicación de técnicas de algoritmos genéticos, lo cual es el caso del modelo implementado en la ciudad de Jaipur (India) (Satsangi, Mishra, Gaur \& Singh, 2003, pp. 808-817).

Un elemento común en la aplicación de la dinámica de sistemas a problemas urbanos es el enfoque en el análisis de los problemas ambientales de las ciudades, valiéndose del potencial de integración sistémica que propicia la metodología (Wee-Kean, Hiroshi \& Yu-Fat, 2009, pp. 1528-1537; Dyson \& Chang, 2005, pp. 669-679; Pfaffenbichler, Emberger \& Shepherd, 2008, pp. 183-200).

Otro ejemplo de la inclusión de esta metodología para analizar las problemas urbanos se da en la Facultad de Arquitectura y Urbanismo de la Universidad de Chile, donde se dicta el curso Modelos de Simulación Urbana, dedicado a comprender la dinámica urbana de la ciudad, concebida como un conjunto de sistemas complejos; la investigación que se desarrolla en el curso se fundamenta en la teoría de sistemas y en la dinámica de sistemas.

Por la importancia de este problema la corporación Electronics Arts ha creado un popular videojuego llamado simCity, el cual simula la dinámica de una ciudad utilizando una mezcla de sistemas de información geográficos y modelos de simulación. Lo sorprendente no es que se haya creado un juego acerca de la dinámica urbana, lo importante es que se ha venido convirtiendo en una herramienta de aprendizaje alrededor del mundo, utilizándose en las aulas de importantes universidades, tal y como se hizo en el curso mencionado.

En ciudades colombianas también se han aplicado modelos de dinámica de sistemas para representar la dinámica urbana, como el modelo creado en la Universidad Tecnológica de Pereira, con el cual se quería estudiar la evolución de la calidad de vida en Pamplona. Para esto se modelaron variables como población, disponibilidad de vivienda y costo de servicios públicos, entre otras (Rueda, 2007). 


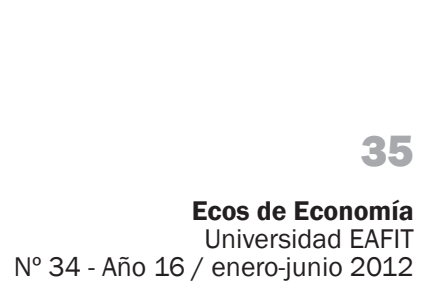

\section{Construcción del modelo}

Para la construcción del modelo lo más importante fue entender el comportamiento de la dinámica urbana en el municipio de Envigado. Para esto se hizo un listado de variables que, se creía en un comienzo, causaban algún efecto en el sistema. Con este listado se comenzó la construcción de un diagrama causal que facilitó el entendimiento del sistema (v. figura 2), para hacer así más sencilla la tarea de construcción del modelo. Vale la pena aclarar que algunas de las variables que aparecen en el diagrama causal no fueron incluidas dentro del modelo debido a que en el proceso de construcción de este no explicaban nada del sistema o no se contaba con información suficiente para hacer una correcta abstracción de la variable, tal y como sucedió con la variable demolición.

Con el objetivo de simplificar la construcción del diagrama causal, se asumió como migración el balance entre la población que deja de vivir en Envigado (emigración) y la población que llega de otras ciudades (inmigración), la cual es muy alta debido a su cercanía con el resto de municipios pertenecientes al área metropolitana de Medellín. También se definió como déficit de vivienda al resultado de la resta entre la oferta de vivienda, ocasionada por la construcción, y la demanda de vivienda ocasionada por la población.

En el diagrama causal se pueden observar ciclos de retroalimentación, tanto positivos como negativos, algunos de los cuales se explicarán a continuación, con la finalidad de lograr una comprensión adecuada del sistema.

Uno de los ciclos positivos más importantes para el modelo es el que relaciona área construida, área sin construir (área sin ningún tipo de infraestructura) y tasa de construcción (ciclo 6). Este ciclo es de gran importancia, ya que es el área que, al transcurrir un tiempo determinado, comenzará a escasear y terminará limitando tanto la construcción de nueva vivienda como el crecimiento de la población. Otro ciclo principal para el modelo es el ciclo 1, que comienza en la variable población y que tras pasar por déficit de vivienda y migración se convierte en un ciclo negativo, lo cual indica que a largo plazo, cuando escasee el área disponible para construcción y por la oferta de nuevas viviendas, no se puedan amortiguar los crecimientos de población y será la migración la encargada de estabilizar el sistema. 
Evaluación de la dinámica urbana para la ciudad de Envigado

ALEJANDRO BETANCOURT

LUIS ANTONIO QUINTERO

Figura 2.

Diagrama que representa la dinámica urbana en el municipio de Envigado

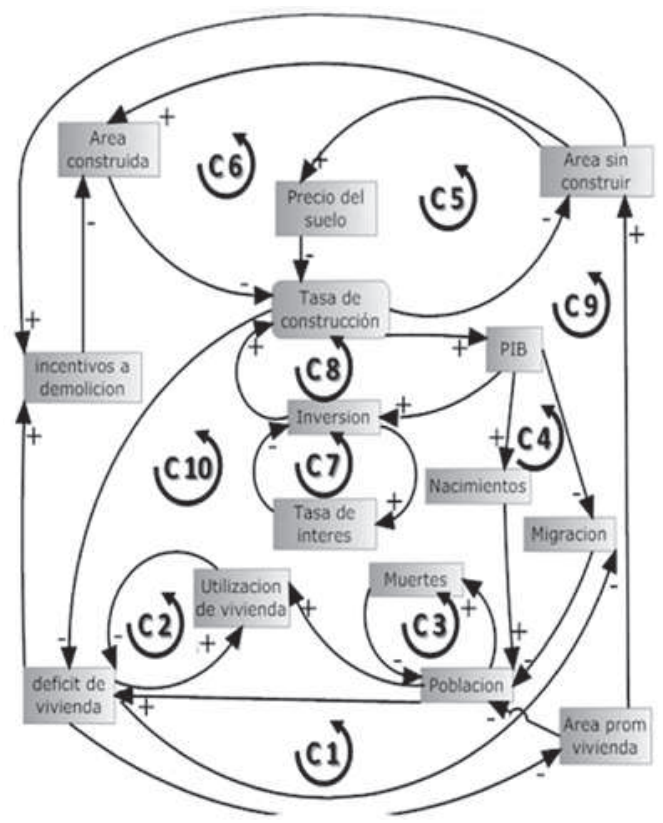

Luego del adecuado entendimiento del sistema, se comenzó a crear el modelo. Para esto fue necesario analizar mucho más en profundidad, algunas de las variables que se encuentran en el diagrama causal. Como resultado de dicho análisis se encontró que la variable más importante del modelo es déficit de vivienda, ya que es esta la que se encarga de resumir el comportamiento que se presenta entre la oferta y la demanda de vivienda, las cuales interactúan entre sí. Para modelar dicha variable se dividió el modelo en dos partes: una se encarga de generar la oferta de vivienda y la otra el crecimiento de la población envigadeña en el transcurso del tiempo. Ambas partes contienen gran cantidad de variables que están sistémicamente interrelacionadas.

Algunas de las variables del modelo son socioeconómicas, como el PIB, la inversión y la dinámica poblacional. Trabajar con este tipo de variables agrega un grado de complejidad mayor al modelo, debido a que son variables de gran fluctuación y por lo tanto es imposible determinar el comportamiento futuro que tendrán. Para solucionar este inconveniente se crearon escenarios que tuvieran en cuenta estas variables y así poder fijar una situación socioeconómica antes de correr el modelo y observar, en ese caso, 
cómo sería la evolución de la ciudad en un periodo determinado, bajo un comportamiento proyectado a partir de estas variables.

No se puede olvidar que el objetivo principal del modelo es apoyar a los gobernantes en la toma adecuada de decisiones, por lo tanto fueron incluidas en el modelo variables que puedan ser modificadas por el usuario para buscar un objetivo específico. Estas variables permiten encontrar puntos óptimos de acuerdo con lo deseado por los gobernantes de Envigado. Ejemplificando un poco, se tiene que si la Oficina de Planeación Municipal se planteara maximizar la cobertura de vivienda para la población, podría correrse el módulo de optimización, estableciendo las variables de monto de inversión en construcción de viviendas y restricciones del POT, para buscar el máximo en cobertura de vivienda.

\section{El modelo}

El modelo fue construido en Powersim y consta de tres partes básicas, dos de las cuales son las encargadas de manejar la oferta y la demanda de vivienda, y la tercera, de tomar esta información y generar los resultados principales, como el déficit de vivienda y los gráficos que resumen la dinámica urbana. Aparte, y como valor agregado al modelo, se construyó una interface gráfica que permite al usuario interactuar más fácilmente y observar los resultados, sin tener que manipular ni entender el funcionamiento de él, esto con el fin de que un usuario sin conocimiento alguno de la dinámica de sistemas pueda operarlo y obtener información útil.

El modelo intercambia información importante con Microsoft Excel, es decir, recibe información de Excel para poder modelar las migraciones y los posibles escenarios del PIB, y además envía los resultados obtenidos para así facilitar el proceso de validación y de análisis de resultados.

\section{Validación}

El hecho de crear un modelo de dinámica de sistemas para cualquier tipo de problema no garantiza la adecuada representación del sistema real, por esto es necesario hacer un proceso de validación con el cual pueda comprobarse la aproximación en el comportamiento del modelo a la realidad. 
Evaluación de la dinámica urbana para la ciudad de Envigado

ALEJANDRO BETANCOURT

LUIS ANTONIO QUINTERO

Para realizar la validación de nuestro modelo se hizo una comparación entre los datos obtenidos por él y los que fueron entregados por la oficina de planeación, estos últimos se encuentran disponibles desde 1998 hasta el 2007.

Con el fin de una adecuada validación del modelo, se estudiaron las tres variables más significativas, que se explican a continuación.

\subsection{Oferta de vivienda}

En el caso de la oferta de vivienda, los datos logrados por el modelo son bastante similares, tanto así que el error promedio es del 0,2\%. En la Figura 3 puede observarse claramente cómo fue el comportamiento de la oferta de vivienda en los años anteriores y cómo el modelo representa ese comportamiento.

Figura 3.

Oferta de vivienda real vs. Oferta de vivienda obtenida por el modelo

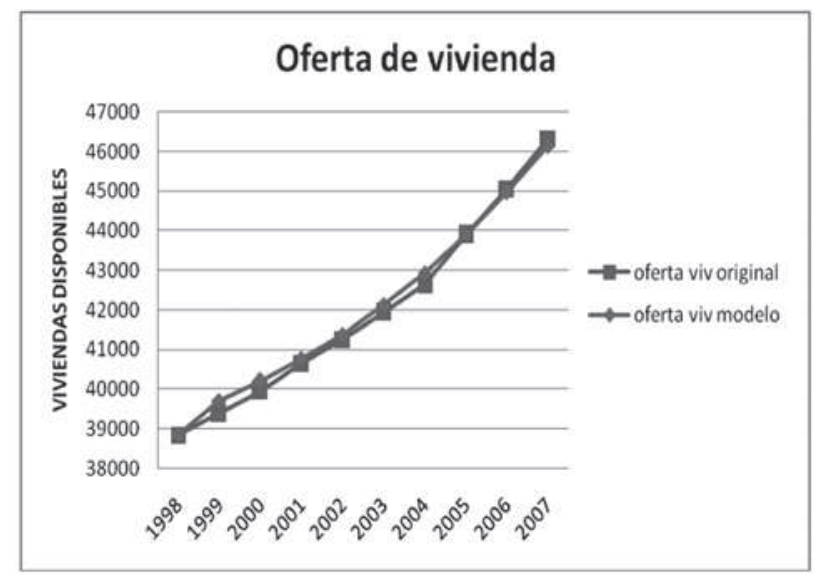

La población total del municipio es un dato de suma importancia para sus gobernantes; sin embargo, se encuentra mucha más información oculta en la población dividida por grupos etarios, ya que es esta la que mostrará qué tan joven será la población del municipio a mediano y largo plazo, por esta razón es relevante que el modelo represente de manera adecuada el comportamiento de la población dividida por edades, para verificar que lo anterior efectivamente sucede. Nótese en las Figura 4 la gran similitud que existe entre la distribución por grupos etarios reales y la generada por el modelo. 
Ecos de Economía

Universidad EAFIT

№ 34 - Año 16 / enero-junio 2012

\section{Figura 4.}

Distribución por edades de la población de Envigado, según el modelo.

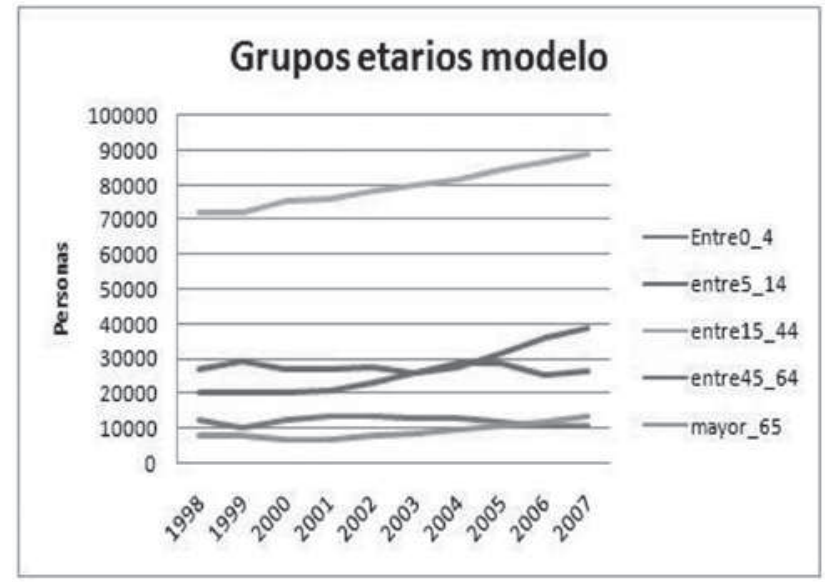

Figura 5.

Distribución real por grupos etarios.

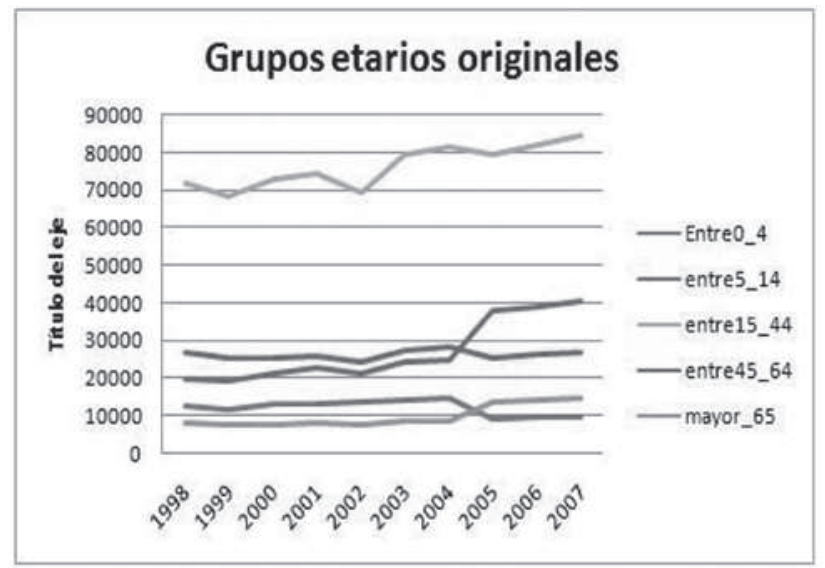

\subsection{Demanda de vivienda}

La demanda de vivienda es ocasionada por el crecimiento de la población; debido a ello es esa evolución la que debe ser estudiada y validada, lo cual puede desarrollarse mediante la población clasificada por grupos de edad o por población total, puesto que esta última es la suma de cada una de las poblaciones por grupos etarios. 
Evaluación de la dinámica urbana para la ciudad de Envigado

ALEJANDRO BETANCOURT

LUIS ANTONIO QUINTERO

El porcentaje de error promedio de este dato es del 1\%, valor que demuestra que la evolución de la población de Envigado en el transcurso del tiempo está bien representada en el modelo. En la Figura 6 puede verse cómo es la evolución de la población, tanto la sugerida por la oficina de planeación, como la representada por el modelo.

Figura 6.

Evolución de la población en el tiempo.

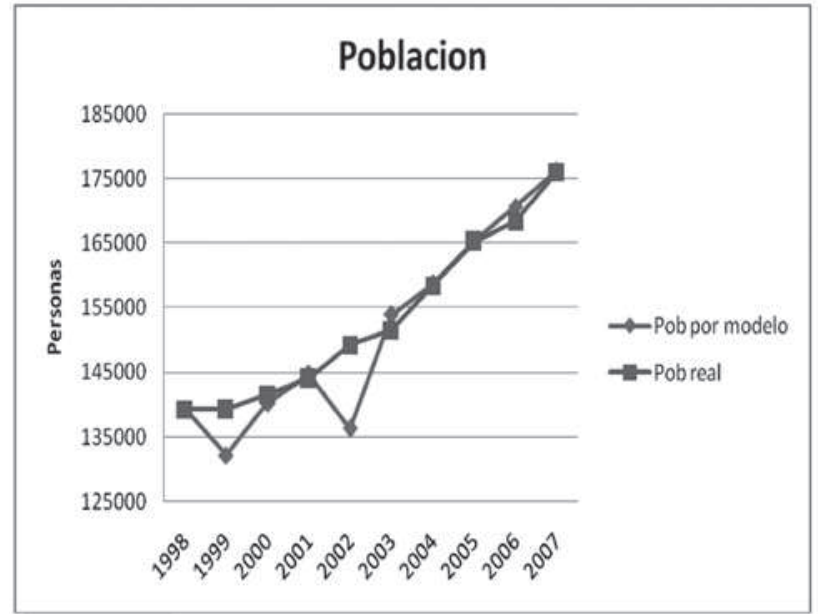

\subsection{Déficit de vivienda}

Luego de haber encontrado que tanto la oferta como la demanda de vivienda son bien representadas por el modelo, es de esperarse que el déficit de ella también lo sea. En la Figura 7 se muestra la evolución del déficit de vivienda en el transcurso del tiempo; para este dato se obtuvo un error promedio del 2,2\%, valor que a pesar de ser un poco más elevado que el encontrado, tanto para la oferta como para la demanda, es bajo y muestra que los valores que toma la variable que resume el modelo son muy acercados a los observados en la realidad. 
Figura 7.

Déficit de vivienda teórico y experimental

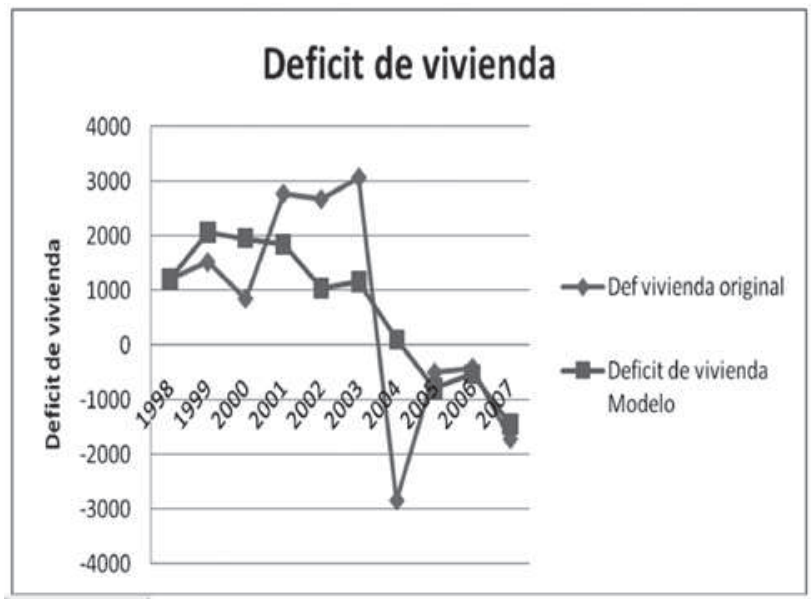

\section{Escenarios y análisis de resultados}

Como ya se dijo, para correr el modelo se necesitan fijar algunas variables que dependen de la situación sociopolítica del país, es decir, seleccionar un escenario en el cual se correrá el modelo y hacer un análisis de los resultados obtenidos. En este trabajo se analizarán algunos de los escenarios que surgen de combinar los diferentes valores posibles que pueden tomar las variables PIB y área promedio de vivienda, indicados en la Tabla 1. Vale la pena aclarar que cada combinación de valores genera un escenario distinto, pero para no perder objetividad en este documento solo se analizarán tres, dos de los cuales se consideran escenarios factibles, y uno de carácter más teórico.

Para comenzar a estudiar los escenarios mencionados se determinó que el PIB seguirá un comportamiento sinusoidal, el cual corresponde a la ocurrencia de ciclos económicos que influyen bastante en la dinámica de la construcción. Dichos ciclos, en este caso, tienen una frecuencia de quince años y una amplitud de aproximadamente cien millones de pesos. Este escenario no es muy factible en la realidad, sin embargo es una forma de mostrar cómo se comporta la dinámica urbana de acuerdo con un concepto teórico.

Al correr el modelo según las condiciones especificadas, se obtuvieron los resultados que se resumen en la Figura 8, en la que se observa el comportamiento de la oferta 
Evaluación de la dinámica urbana para la ciudad de Envigado

ALEJANDRO BETANCOURT

LUIS ANTONIO QUINTERO

Tabla 1.

Posibles valores de las variables PIB y área promedio de vivienda (APV)

para generar los escenarios

\begin{tabular}{|c|c|}
\hline PIB & APV \\
\hline sinusoidal & \multirow{2}{*}{$\begin{array}{c}\text { entre } 90 \mathrm{Mt} t^{\wedge} 2 y \\
105 \mathrm{Mt} \mathrm{t}^{\wedge} 2\end{array}$} \\
\hline constante & \\
\hline $\begin{array}{l}\text { levemente } \\
\text { creciente }\end{array}$ & \multirow{2}{*}{$\begin{array}{c}\text { entre } 105 \mathrm{Mt} t^{\wedge} 2 y \\
120 \mathrm{Mt} t^{\wedge} 2\end{array}$} \\
\hline creciente & \\
\hline $\begin{array}{c}\text { muy } \\
\text { creciente }\end{array}$ & \multirow{2}{*}{$\begin{array}{c}\text { Entre } 120 \mathrm{Mt}^{\wedge} 2 y \\
150 \mathrm{M} \mathrm{t}^{\wedge} 2\end{array}$} \\
\hline decreciente & \\
\hline
\end{tabular}

y la demanda de vivienda en el transcurso del tiempo y con la cual se puede obtener información de gran importancia acerca de la dinámica urbana que se presentaría en el municipio de Envigado, como lo es, por ejemplo, el déficit promedio de vivienda (las simulaciones indican 6.021 viviendas sin habitar, y el punto de saturación de la población del municipio, que comienza a ser alcanzado en el año 2040 y que muestra cuándo la población empieza estabilizarse y a alcanzar el valor máximo (316.000 habitantes), y cuándo comenzarán a escasear las tierras disponibles para vivienda en el municipio (89.478 viviendas). Esta información se despliega en la figura 8.

En la Figura 9 se muestra cómo será la distribución por edades de la población de Envigado. Se nota claramente que es una población joven y en edad de trabajar, ya que en su mayoría la componen personas entre 15 y 45 años, y en su minoría, entre 65 y 100 años.

Por otra parte, dejando un poco atrás este escenario teórico, se procede a estudiar un escenario en el cual se supone un PIB muy creciente y una política de planeación que no permita la construcción de viviendas mayores de 105 m2, valores que se encuentran por debajo del tamaño de vivienda promedio del municipio, el cual, con datos hasta el 2007, es de 116 m2. 
Ecos de Economía

Universidad EAFIT

№ 34 - Año 16 / enero-junio 2012

Figura 8.

Demanda de vivienda contra oferta de vivienda con un PIB sinusoidal.

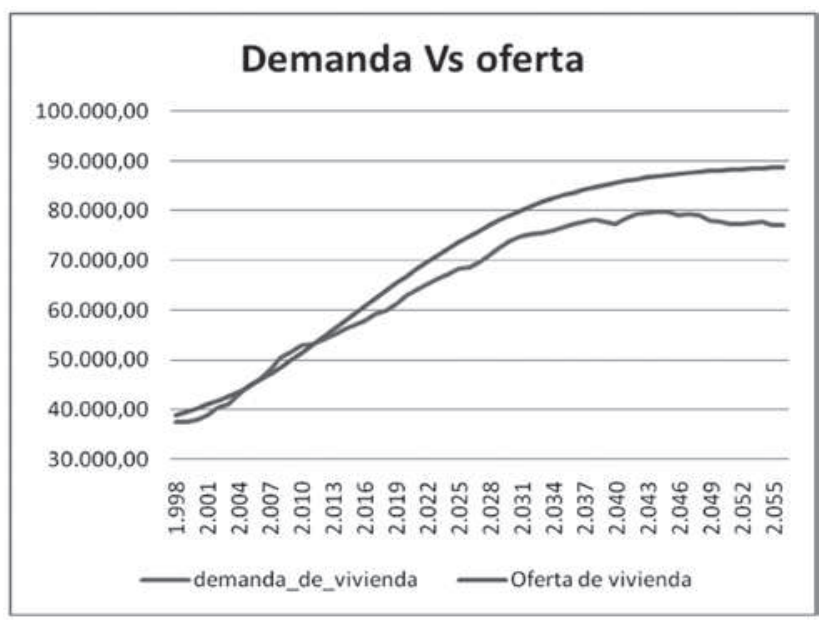

Figura 9.

Población dividida por grupos etarios bajo un PIB.

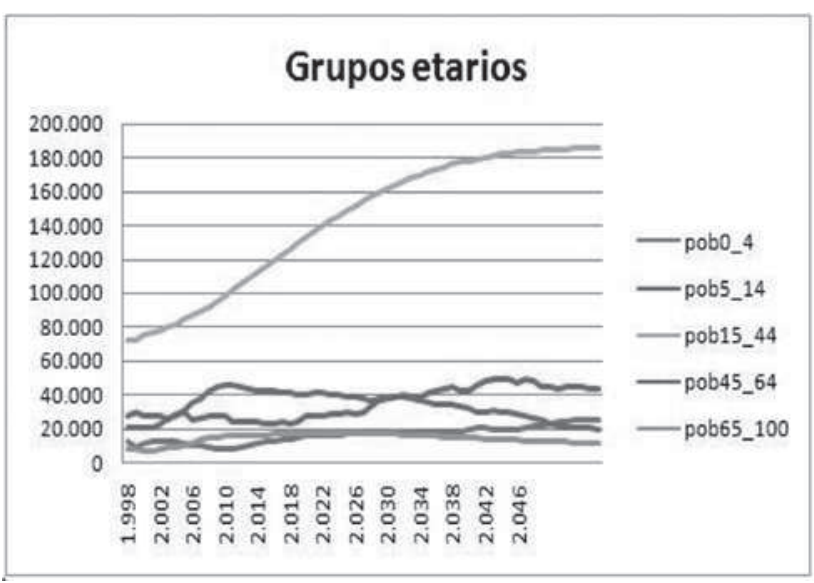

En este caso el punto de saturación se alcanza aproximadamente en el año 2040 (v. Figura 10), llegando a un máximo de 350.000 habitantes, con aproximadamente 92.373 viviendas disponibles y un déficit promedio de 2.450 viviendas. 


\section{4}

Evaluación de la dinámica urbana para la ciudad de Envigado

ALEJANDRO BETANCOURT

LUIS ANTONIO QUINTERO

\section{Figura 10.}

Demanda de vivienda contra oferta de vivienda bajo un PIB creciente y una política de planeación de casas pequeñas.

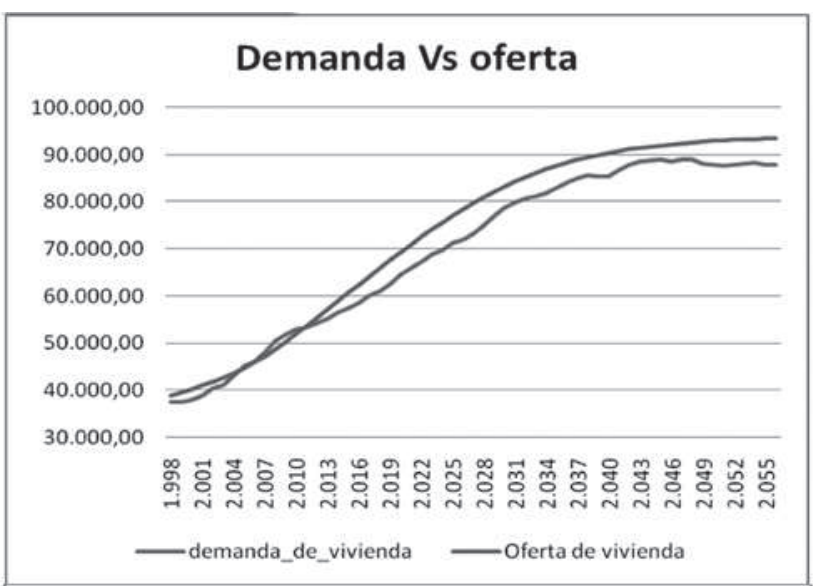

En este escenario la población con edades altas es mucho más grande que en el caso anterior, ya que el número de personas entre 45 y 100 años pasó de ser un grupo de poco peso, a otro con peso significativo (v. Figura 11).

Figura 11.

Población dividida por grupos etarios bajo un PIB muy creciente y una política de planeación de casas pequeñas

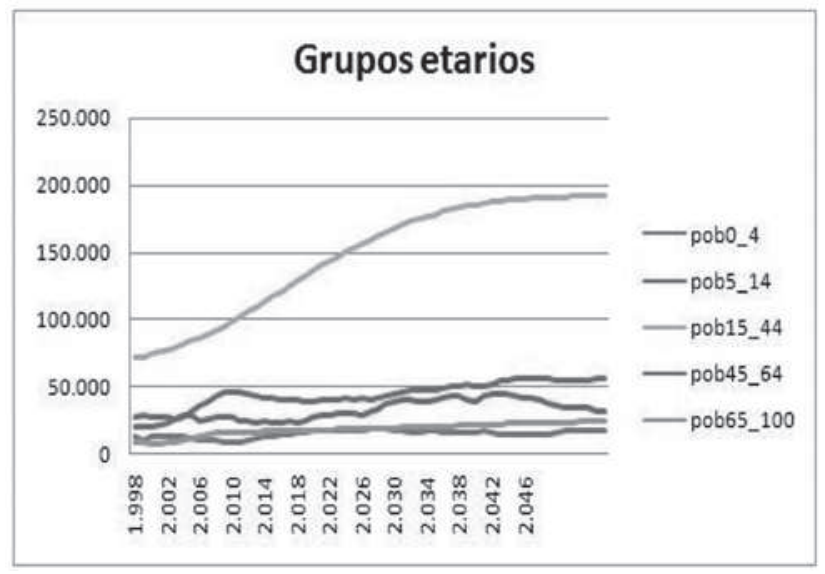


Ecos de Economía

Por último, se estudiará la situación de Envigado en el caso de un PIB muy creciente y una política de planeación que permita viviendas entre 120 m2 y 150 m2. En la Figura 12 se resume la dinámica urbana para este escenario, en el cual, el punto de saturación es alcanzado cerca del año 2046, llegando a un máximo de 81.030 viviendas disponibles, 80.083 viviendas demandadas y un déficit promedio de 2.884 viviendas sin ser habitadas.

Figura 12.

Demanda vs. oferta para un PIB creciente y una política de planeación de casas grandes.

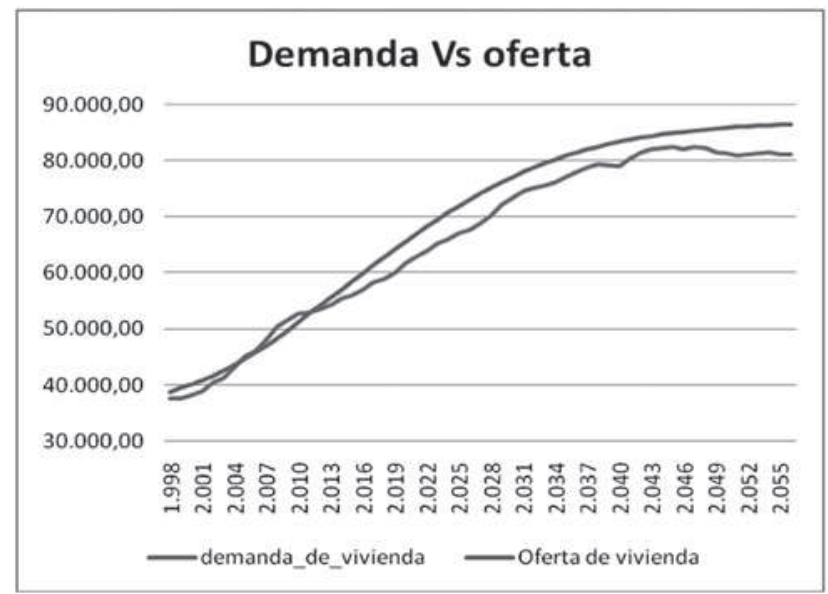

Nótese en la Figura 13 que en este escenario la población adulta de Envigado es mucho más significativa que en los escenarios anteriores, a pesar de que la población máxima obtenida en este caso, de 327.431 personas, es menor que en el caso anterior: 375.000 personas.

Luego de haber corrido dos escenarios en las mismas condiciones del PIB, y de cambiar las políticas de planeación, se podría tomar una decisión más segura para la búsqueda de un fin determinado. Por ejemplo, si los gobernantes creen que lo mejor para Envigado en un futuro es una población pequeña y de edades altas, y si fuese el caso de un PIB muy creciente, como el de los últimos años, lo que deberían hacer es otorgar permisos de construcción solo para viviendas mayores de 120 m2. 
Evaluación de la dinámica urbana para la ciudad de Envigado

ALEJANDRO BETANCOURT

LUIS ANTONIO QUINTERO

Figura 13.

Grupos etarios en un escenario con un PIB muy creciente y una política de planeación de casas grandes.

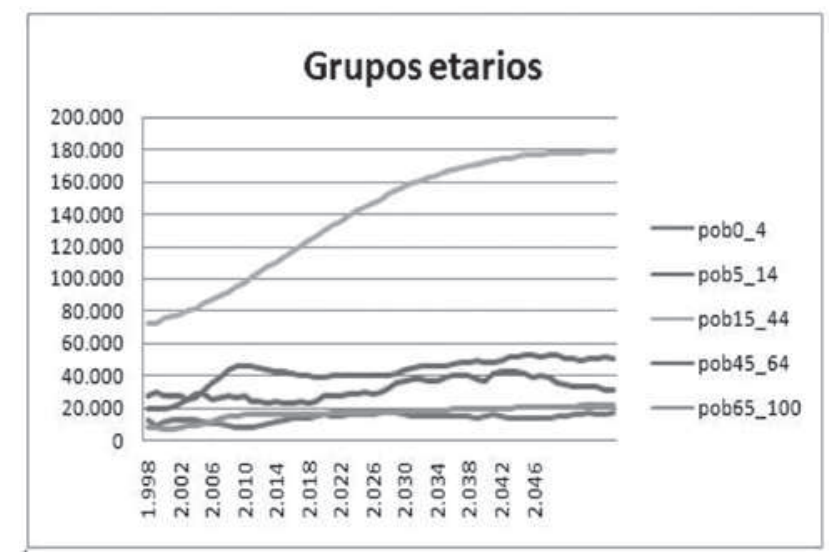

\section{Conclusiones y recomendaciones}

Este trabajo resultará de gran importancia como herramienta para la toma de decisiones en las diversas oficinas del municipio, ya que les permitirá ver las repercusiones de las decisiones que se tomen en un momento determinado; además, gracias a que trata un problema que es de gran preocupación en las ciudades, podría ser aplicado en otros municipios que conforman el Área Metropolitana del Valle de Aburrá, lo cual permitiría a las administraciones correspondientes gestionar recursos que ofrecen el gobierno y los municipios para el desarrollo de vivienda urbana.

La implementación de este tipo de modelos por parte de instituciones del orden municipal ayudaría al buen logro de los objetivos, mediante un apoyo para la planeación y la gestión de los problemas que trae consigo el crecimiento de las ciudades.

\section{Referencias}

Colombia, Departamento Administrativo Nacional de Estadística (DANE). Recuperado el 20 de junio de 2008, de http://www.dane.gov.co

Chunyang, H.; Norio, O.; Qiaofeng, Z.; Peijun, S. \& Jingshui, Z. (2006, pp. 323-345). Modeling urban expansion scenarios by coupling cellular automata model and system dynamic model in Beijing, China. Applied Geography, 323-345. 
Corporación otra parte (s. f.). Recuperado el 10 de junio de 2008, de http://www.otraparte.org

Durán-Encalada, J.A. \& Paucar-Cáceres, A. (2009). System Dynamics Urban Sustainability Model for Puerto Aura in Puebla, Mexico. Syst Pract Action Res., 77-99.

Dyson, B. \& Chang, N.-B. (2005). Forecasting municipal solid waste generation in a fastgrowing urban region with system dynamics modeling. Waste Management, 25, 669-679.

Forrester, J.W. (1969). Urban Dynamics. Cambridge, Mass.: MIT Press.

Gray, J.M.; Ressel, D. \& Vararja, P.P. (1972, abril). A Critique of Forrester's Model of an Urban Area. IEEE Trans. Systems, Men and Cybernetics, SMC-2, (2), 139-144.

Guan, D.; Gao, W.; Su, W.; Li, H. \& Hokao, K. (2011). Modeling and dynamic assessment of urban economy-resource-environment system with a coupled system dynamics geographic information system model. Ecological Indicators, 11, 1333-1344.

Harveya, G. \& Roberth, W. (1970, diciembre). A Critical Look at Urban Dynamics: The Forrester Model and Public Policy. Washington, D.C.: Urban Institute.

Ingram, G.K. \& Kain, J.F. (1972, enero). Two Views of Urban Dynamics. Discussion Paper Series. Harvard, EE.UU.: Harvard Institute of Economic Research, (228).

Kollikkathara, N. (2009). Integrated system Dynamic study and prognosis on municipal solid waste management for northern New Jersey Urban Area. [A dissertation for degree of doctor of Environmental Management]. New Jersey, EE.UU.: Montclair State University.

Moonseo, P.; Youngjoo, K.; Hyun-Soo, L.; Sangwon, H.; Sungjoo, H. \& Min, J.Ch. (2011, 31 de mayo). Modeling the dynamics of urban development project: Focusing on selfsufficient city development. Mathematical and Computer Modelling, 1-12.

Pfaffenbichler, P.; Emberger, G. \& Shepherd, S. (2008). The integrated dynamic land use and transport model MARS. Network Spatial Economics, 8, 183-200.

Reverté Calvet, G. (2003, diciembre). Proyecciones demográficas para la ciudad de Mataró. Boletín de Dinámica de Sistemas (BADS). 
Evaluación de la dinámica urbana para la ciudad de Envigado

ALEJANDRO BETANCOURT

LUIS ANTONIO QUINTERO

Rueda, L.J. (2007, mayo). Moldeamiento inicial de ciudades de países en vía de desarroIlo, utilizando dinámica de sistemas. Scientia et Technical, XIII, (34).

Satsangi, P.S.; Mishra, D.S.; Gaur, S.K. \& Singh, B.K. (2003, pp. 808-817). Systems Dynamics Modelling simulation and optimization of integrated urban systems: a soft computing approach. Kybernetes, 32, (5-6), 808-817.

Saxton, D.E. (1979, enero). Evaluating urban growth policies with a systems simulation. Management Science, 25, (1), 43.

Secretaría de Planeación de Envigado (2007), Anuario estadístico del municipio de Envigado, s. d.

Wee-Kean, F.; Hiroshi, M. \& Yu-Fat, L. (2009). Application of System Dynamics model as decision making tool in urban planning process toward stabilizing carbon dioxide emissions from cities. Building and Environment, 44, 1528-1537. 